\title{
Impfungen
}

\section{Pneumokokken-Erkrankungen verhindern}

_ Die Gefahr von Infektionen mit Pneumokokken wird immer noch unterschätzt, mahnte Prof. Jörg Schelling, Martinsried. Invasive Pneumokokken-Erkrankungen führen immerhin bei 30-40\% der Erwachsenen ab 65 Jahren zum Tod.

„Eine Lungenentzündung ist keine leichte Erkrankung“, betonte Prof. Adrian Gillissen, Kassel. Jeder fünfte Patient mit ambulant erworbener Pneumonie

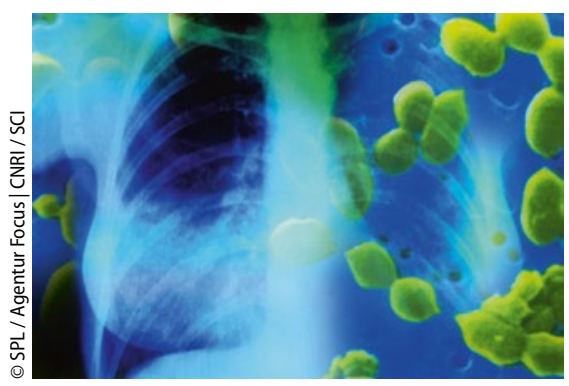

Pneumokokken bedrohen unter anderem auch die Lunge. muss ins Krankenhaus, und bei 17\% der hospitalisierten Patienten, die gleichzeitig Komorbiditäten wie Herzerkrankungen, Diabetes u. a. aufweisen, führt die Pneumonie zum Tod. „Diese Patienten sollten geimpft werden“, riet Gillissen.

\section{Konjugatimpfstoff immunisiert auch Ältere}

Die STIKO empfiehlt die Impfung gegen Pneumokokken-Erkrankungen für Säuglinge und Kleinkinder bis zwei Jahre, Erwachsene ab 60 Jahren, und darüber hinaus für alle Personen, bei denen eine erhöhte gesundheitliche Gefährdung besteht. Ab dem 5. Lebensjahr kann für diese Indikationsimpfung sowohl ein Polysaccharidimpfstoff als auch der 13-valente Konjugatimpfstoff verwendet werden.

Dass auch ältere Menschen ab 65 Jahren von dem 13-valenten Konjugatimpf- stoff Prevenar $13^{\circledast}$ profitieren, bestätigt die CAPiTA-Studie (Community-Acquired Pneumonia Immunization Trial in Adults) [Bonten M et al. N Engl J Med. 2015]. In dieser randomisierten, placebokontrollierten Studie mit rund 85.000 erwachsenen Teilnehmern zeigte sich, dass die Häufigkeit von ambulant erworbenen Lungenentzündungen, die durch einen im Impfstoff enthaltenen Serotyp ausgelöst wurden, durch die Impfung um $45 \%$ reduziert werden kann. Impfstoff-Serotyp-bedingte invasive Pneumokokkenerkrankungen gingen sogar um $75 \%$ zurück. Schelling erinnerte daran, dass es wichtig sei, die Patienten auf die empfohlene Impfung aufmerksam zu machen.

\section{Dr. Judith Neumaier}

- Fachpressegespräch „Prävention von Pneumokokken-Pneumonie: Die Bedeutung der Impfung gegen Pneumokokken und die Besonderheiten des Konjugatimpfstoffs Prevenar $13^{\Phi " ;}$; München, Oktober 2015 (Veranstalter: Pfizer)

\section{Typ-2-Diabetes}

\section{Therapieziel Nüchternglukose $<100 \mathrm{mg} / \mathrm{dl}$ ohne Hypoglykämie}

_ Basisinsulin ist ein Grundbaustein der Diabetestherapie. Wenn beim Typ 2 die Insulintherapie mit einer basal unterstützten oralen Therapie (BOT) beginnt, hat dies meist eine deutliche Verbesserung des Glukosestoffwechsels zur Folge. Allerdings sollte das Basalinsulin so titriert werden, dass ein normaler Nüchternblutzucker von $<100 \mathrm{mg} / \mathrm{dl}$ bzw. $<5,6$ $\mathrm{mmol} / \mathrm{l}$ erreicht wird, erklärte Prof. Thomas Forst, Neuss. Denn eine gute Einstellung entlastet die Betazellen und gewährleistet eine gute Insulinantwort nach den Mahlzeiten.

\section{Die Blutzuckereinstellung - immer ein Balanceakt}

Die normnahe Einstellung der Nüchternglukose setzt voraus, dass das Basalinsulin gleichmäßig über 24 Stunden wirkt und dass diese Wirkung konstant einsetzt. Dies ist bei Neutral-Protamin-
Hagedorn(NPH)-Insulinen nicht der Fall, so Forst: Die instabile Absorptionskinetik sorgt für schwankende Insulinspiegel über den Tag, von Tag zu Tag und von Patient zu Patient. Arzt und Patient müssen entweder häufige Unterzuckerungen oder eine nicht so strikte Blutzuckereinstellung in Kauf nehmen.

Langwirksame Analoginsuline wie Insulin glargin oder Insulin detemir besitzen im Vergleich zu NPH eine flacheres Wirkprofil im 24-Stunden-Verlauf. Mit ihnen gelingt bei gleich guter Blutzuckerkontrolle eine signifikante Reduktion des Hypoglykämie-Risikos.

\section{Stabile Freisetzungskinetik}

Einen weiteren Fortschritt ermöglicht eine veränderte Formulierung von Insulin glargin (U100), die als U300 (Toujeo ${ }^{\circledast}$ ) seit dem Frühjahr 2015 verfügbar ist. Bei U300 liegt das Insulin dreifach konzen- triert vor, was zu einer Halbierung der Absorptionsoberfläche führt. Die Folgen: Längere Halbwertszeit, geringere Absorptionsvariabilität und eine gleichmäBigere Wirkung über 24 Stunden.

In Vergleichsstudien mit Insulin glargin zeigte U300 bei gleich guter Blutzuckereinstellung ein signifikant reduziertes Risiko für Hypoglykämien: Über 24 Stunden war es um $14 \%$ reduziert, nachts um 31\%. Gerade die Reduktion des Risikos für nächtliche Hypoglykämien ist laut Forst wichtig, da diese eine erfolgreiche Titration behindern. Zudem essen Diabetiker mehr, wenn nächtliche Unterzuckerungen auftreten. Dies erklärt, warum Patienten unter U300 etwas weniger Gewicht zunahmen als unter U100.

Dr. Dirk Einecke

- Pressekonferenz "Highlights Sanofi Diabetes 2015: Mit der richtigen Einstellung die Ziele erreichen"; Berlin, Dezember 2015 (Veranstalter: Sanofi) 